\title{
Conversations avec Edmund Husserl (1931-1936)
}

Adelgundis Jaegerschmid et O. S. B.

Traducteur : Julien Farges

\section{OpenEdition}

\section{Journals}

Édition électronique

URL : https://journals.openedition.org/alter/2225

DOI : $10.4000 /$ alter.2225

ISSN : 2558-7927

Éditeur :

Association ALTER, Archives Husserl (CNRS-UMR 8547)

Édition imprimée

Date de publication : 1 novembre 2020

Pagination : 321-350

ISBN : 978-2-9550449-6-4

ISSN : 1249-8947

\section{Référence électronique}

Adelgundis Jaegerschmid et O. S. B., "Conversations avec Edmund Husserl (1931-1936) », Alter [En

ligne], 28 | 2020, mis en ligne le 22 décembre 2020, consulté le 12 décembre 2021. URL : http:// journals.openedition.org/alter/2225; DOI : https://doi.org/10.4000/alter.2225

Ce document a été généré automatiquement le 12 décembre 2021.

Revue Alter 


\section{Conversations avec Edmund Husserl (1931-1936)}

Adelgundis Jaegerschmid et O. S. B.

Traduction: Julien Farges

\section{Avant-propos du traducteur}

1 Nous proposons ci-après la traduction du célèbre témoignage que la sœur bénédictine Adelgundis Jaegerschmid a publié en 1981 dans le $n^{\circ} 199$ de la revue Stimmen der Zeit (p. 48-58 et 129-138). Pour avoir été cités ici ou là, quelques extraits de cette chronique, en particulier quelques propos remarquables de Husserl, sont déjà connus des lecteurs francophones. Mais la présente traduction permet de les ressaisir dans ce qui fut leur contexte d'origine, à savoir la relation tout à fait exceptionnelle qui s'est nouée au fil des années entre Husserl et sœur Adelgundis et qui s'est développée finalement sous la forme d'une unique et vaste conversation où, au-delà des aspects strictement biographiques, c'est de la religion en général, de la foi chrétienne en particulier et de leur articulation avec la philosophie et la phénoménologie qu'il est constamment question.

2 Signalons qu'une traduction anglaise de ce texte a été publiée par Marcus Brainard sous le titre unique "Conversations with Edmund Husserl, 1931-1938" (in The New Yearbook for Phenomenology and Phenomenological Research, 1, 2001, p. 331-350), dont nous avons retenu deux choix: nous avons réduit l'usage fréquent que sœur Adelgundis fait des italiques (notamment pour signaler les concepts qui forment le thème principal de chaque conversation) au seul soulignement marquant l'insistance du propos, et nous avons substitué ses initiales (AJ) à la mention « moi » lorsqu'elle rapporte ses propres paroles.

3 Les indications entre parenthèses sont des précisions fournies par sœur Adelgundis; tout ce qui figure entre crochets est ajout du traducteur, de même que toutes les notes infrapaginales.

4 Nous adressons nos remerciements à l'éditeur Herder pour nous avoir autorisé à publier cette traduction - et à Marguerite Léna sfx, pour sa relecture et ses suggestions.

5 Julien Farges 
[48] Durant les dernières années de la vie d'Edmund Husserl, alors que les premiers signes de la tragédie nationale-socialiste étaient déjà visibles, j'ai gardé la trace de nos conversations sous la forme d'un journal, rédigé sur des feuilles volantes immédiatement après chacune de nos entrevues, à Fribourg et parfois même dans le tramway qui me ramenait au couvent Sainte Lioba à Günterstal. Comme historienne chevronnée, j'ai sobrement pris acte du fait que je n'étais qu'un intermédiaire, le traditor de ce que Husserl me communiquait de sa vie intérieure personnelle dans une belle confiance humaine. Je voulais sauvegarder ses paroles pour une époque à venir.

7 En septembre 1938, cinq mois après le décès de Husserl, sa veuve Malvine m'adressa le père franciscain belge H. L. van Breda (le fondateur des Archives Husserl à Louvain), qui était à la recherche d'informations sur la personne de Husserl pour sa thèse de doctorat. Au vu de ce qu'avait d'inquiétant le contexte politique mondial à l'époque, le père van Breda m'a incitée catégoriquement à taper ces souvenirs le soir même. Ces souvenirs sont un document historique, une ressource modeste, dépourvue de tout vernis littéraire - et ne souhaitent pas non plus être autre chose.

\section{8 avril 1931}

10 Visite en soirée, durant presque deux heures. J'essaie très rapidement de faire en sorte que ce soit Husserl qui mène la conversation. J'émets parfois des objections et le force ainsi à clarifier certains problèmes difficiles.

11 «La vie monastique, la vie religieuse chrétienne en général, se déroule toujours sur le fil du rasoir. Elle chute aisément, mais se relève toujours. Elle n'a qu'un but: voir le monde en Dieu, mais elle ne nie pas le monde. Le risque est alors, bien sûr, que l'âme s'enracine par trop dans le monde ou bien que la charité active ainsi que la piété deviennent de simples routines ".

12 Il parle ensuite de la religion indienne. Il me recommande chaleureusement le livre de Romain Rolland sur Gandhi ${ }^{1}$, qu'il venait de lire. «Par opposition au christianisme, la religion indienne a le Nirvana; elle nie le monde. Toute activité suscite de la passivité et court ainsi le risque de la stagnation. Mais toute passivité - le repos en Dieu conçu comme aboutissement - requiert de nouveau l'activité : la charité active ». - AJ : «C'est exactement ce que dit Thomas ». - Husserl : « Oui, tous les grands hommes de la Terre parlent ainsi. Toute résolution est déjà une activité de la volonté. Tout ce qui est acquis comme un résultat de l'activité produit de la passivité et, par conséquent, un risque. Ce qui signifie donc que tout ce qui a été acquis doit toujours être réactivé ».

[49] Nous parlons de la vie religieuse et de la vocation [Berufung] à une vie au sein d'un ordre. AJ : "Pour vivre la vie religieuse, il faut en avoir la vocation [berufen]». Husserl : «Mieux : il faut y avoir été appelé [gerufen]. Cela relève purement de la grâce. Je n'ai pas accès à cette sphère, bien que j'aie toujours été l'un des plus fervents chercheurs de Dieu depuis ma jeunesse. La science authentique est honnête et pure; elle a l'avantage de la véritable humilité, et pourtant, elle a en même temps la capacité d'être critique et de faire des distinctions. De nos jours, le monde ne connaît plus la science véritable; elle est tombée dans la spécialisation la plus étroite. C'était différent à notre époque. L'amphithéâtre était notre église et les professeurs étaient les prédicateurs ».

14 Je lui racontai comment, durant notre jeunesse, nous avions cherché à l'université la science authentique au-delà des examens et de la question des moyens de subsistance, 
et comme nous l'avions servie dans un pur enthousiasme. Bien sûr, seuls quelques-uns, en très petit nombre, adoptaient un point de vue qui visait par-delà les examens. J'ai alors conclu en disant : «Il nous est arrivé, à nous aussi, de brûler pour la science. Mais comment pensez-vous que la science puisse sauver notre monde et l'élever davantage ? Car ce n'est toujours que pour quelques-uns qu'elle est disponible ».

Husserl: «La science authentique rend désintéressé et bon. Aujourd'hui, même les savants intégralement matérialistes ou naturalistes (les savants dans les sciences naturelles) peuvent consacrer leur vie à leur science, même les mathématiciens chez qui on ne trouve pas une once de foi. Dans ces conditions, la science est bonne même si elle ne conduit pas à la religion. D'un autre côté, pourtant, il est impossible de prétendre qu'une science conduisant ultimement à la religion et à Dieu ne soit pas une science authentique. La pédagogie, sous toutes ses formes (pas seulement à l'école) doit porter plus loin les résultats de la science authentique et les mettre en pratique de sorte que le monde en soit renouvelé. C'est ce que vous devez faire, sœur Adelgundis ; la détresse de l'âme est grande. Ce qu'il y a de meilleur sera toujours l'amour - l'amour authentique et pratique de son prochain, qui est fondé dans l'amour de Dieu, lequel ne se trouve pas toujours au sein des confessions. Il arrive souvent que la religion soit discréditée parce que les personnes religieuses ne sont pas du tout intérieurement religieuses. Il s'agit si souvent d'une simple apparence, si souvent de convention et de superstition!

16 "La science authentique doit être science universelle, embrassant la totalité des évidences sur le fondement de l'autonomie, totalité au sein de laquelle la religion est elle aussi incorporée. Le christianisme a sa place dans cette sphère. En partant de cette science universelle telle que la phénoménologie l'a élaborée, on parvient finalement à un développement théologique qui conduit ultimement à Dieu, à l'absolu ».

En réponse à ma question de savoir s'il croyait donc réellement en l'absolu (il l'avait dénié précédemment), il me dit: "Ce sont là des relativités, et nous devons avoir le courage de voir ces relativités en face. Elles peuvent elles aussi être des évidences ; par exemple : pour des peuples primitifs, la logique contient des évidences complètement différentes des nôtres. En dernier ressort, nous pouvons restreindre notre perspective et comprendre cet état de fait; nous pouvons nous y plonger en pensée. C'est bien aussi de cette façon que je vis dans ma conscience [50] la douleur d'autrui sans en faire l'expérience dans mon propre corps. La phénoménologie comme science est là pour ceux qui n'ont pas d'accès à la foi comme celui que vous avez. Que peuvent faire tous ceux qui ne rencontrent la religion que tard dans la vie ? Ils ne parviennent plus à entrer dans une relation personnelle avec elle».

J'étais intéressée par la question des rapports entre liturgie et phénoménologie, puisqu'après tout, la liturgie est un opus operatum ${ }^{2}$ et peut figurer quelque chose comme une réduction phénoménologique. Husserl n'a pas su quoi répondre à cela ; pour lui, l'efficacité religieuse n'est pensable qu'au travers des individus et par la possibilité de revivre leur sainteté. Il a une certaine familiarité avec Thérèse d'Avila qu'il a un peu étudiée lorsque, poussée par les écrits de Thérèse, Edith Stein est entrée dans l'Église catholique. Husserl comprend toujours Thomas et les mystiques - pour autant qu'ils sont une expression et un précipité de la vie religieuse - comme des extériorisations subjectives de la vie religieuse. En réponse à mon objection selon laquelle c'est précisément dans son objectivité (comme sacrement) que la liturgie s'accommode avec la phénoménologie, Husserl dit : 
19 «L'erreur la plus dangereuse consiste à croire que c'est par la vérité objective que les erreurs subjectives doivent pouvoir être surmontées de la meilleure façon. Non, seul un subjectivisme plus poussé peut surmonter le subjectivisme, pour autant qu'on le prenne totalement au sérieux et qu'on ne se fasse pas d'illusions sur lui ».

Il évoque ensuite Karl Barth et me montre un numéro de la revue Zwischen den Zeiten. Nous parlons également de Dietrich von Hildebrand et d'Edith Stein, dont le cheminement l'émeut particulièrement: "Les gens peuvent continuer à être personnellement attachés les uns aux autres même si leurs visions du monde les ont séparés. C'est ce qu'Edith a démontré après sa conversion. À l'opposé, après la sienne, Hildebrand s'est détourné. De façon assez curieuse, un certain nombre de mes étudiants se sont décidés radicalement pour la religion et de fait, certains furent ou devinrent des protestants profondément croyants, tandis que d'autres se sont convertis au catholicisme. Rien ne s'est trouvé changé par là dans leurs relations avec moi ; elles sont toujours portées par une confiance mutuelle. De plus, je suis toujours disponible pour débattre de la vérité de façon approfondie. Je suis toujours prêt à reconnaître mes erreurs et à me soumettre ».

21 Au moment de mon départ, nous parlâmes de l'Ancien et du Nouveau Testament. Il me montra son bureau, sur lequel se trouvait la Bible, et il me dit : « Qui est encore capable de saisir l'Ancien Testament? En ce moment, les prophètes Jérémie et Isaïe sont ceux que je préfère. À une époque, pendant ma jeunesse, je ne fus plus capable du tout de comprendre l'Ancien Testament. Il me paraissait si dépourvu de sens; mais c'est loin d'être le cas, n'est-ce pas?»

\section{5 décembre 1933}

Husserl : « Le martyre est le principe de l'Église. Il me semble que c'est le point unique sur lequel elle doit de nouveau réfléchir. Mais reçoit-elle encore le soutien du peuple aujourd'hui, de telle sorte qu'elle puisse le conduire à un nouveau "combat pour la culture $»^{3}$ ? Ou bien n'ose-t-elle plus s'engager dans une telle bataille ? L'Église n'auraitelle pas mieux fait de ne pas choisir le plus grand de [51] deux maux et de renoncer et à signer le Concordat avec l'Allemagne ${ }^{4}$ ? Pour la science également, le martyre deviendra le seul chemin de salut possible. Seuls des héros de l'esprit, des humains radicaux, peuvent peut-être sauver une nouvelle fois la science. L'Église a toujours représenté la rationalité par la synthèse de la raison et de la foi. Mais bien trop de personnes n'étaient qu'extérieurement religieuses; assurément, elles croyaient l'être intérieurement et être fermement assurées dans leur foi catholique, mais elles sont aujourd'hui en pleine faillite! De même, la science ne faisait plus l'objet d'une préoccupation intérieure pour les gens ; sinon, ils ne l'auraient pas jeté par-dessus bord comme ils le font à présent, avec le cœur aussi léger ! Il y aura aussi des martyrs dans la science. Car quiconque veut s'engager aujourd'hui en faveur de la science pure doit avoir le courage de devenir un martyr.

« Voyez-vous, l'Église et la science ont le même but: Dieu. Les uns l'atteignent sur le chemin de l'adoration et de la caritas, les autres sur le chemin de la recherche spirituelle et d'une vie morale. Mais les deux voies sont menacées par le scepticisme et la sophistique, sous quelque forme que ce soit. Cela a conduit l'Église à se faire trop politique, trop enracinée ici-bas ; la science a sombré dans le matérialisme et dans un rationalisme sans fondement. Les conséquences s'en font sentir aujourd'hui dans la distorsion et la mystification de tous les ordres quels qu'ils soient ». 


\section{3 février 1934}

Husserl : « La valeur d'un chrétien se décidera toujours lorsqu'il sera capable de devenir un martyr. Mais beaucoup d'entre vous (les catholiques) considèrent la vie spirituelle comme un billet gratuit pour un fauteuil de premier rang au paradis, pour assister à une première avec louanges et encens. Si la Réforme, la plus grande des bénédictions pour l'Église catholique qui avait tant besoin de réforme, a connu un succès si aisé et si rapide, c'est en raison de l'inculture grossière qui était celle du clergé. Aujourd'hui, le clergé a assurément beaucoup étudié, il est bien instruit en latin, en grec et dans d'autres domaines, mais il y a trop d'occupations au séminaire et trop peu de vie personnelle responsable, trop peu d'expérience du divin dans l'individu. - Une question: Edith Stein devait pourtant bien avoir une connaissance suffisante du caractère pour le moins nuancé de l'engagement spirituel qui était celui de la scolastique. Comment se fait-il qu'on ne trouve pas la moindre trace d'une telle restriction chez sainte Thérèse d'Avila?» - $\mathrm{AJ}:$ "C'est sans aucun doute un mystère pour ceux qui considèrent la chose de l'extérieur : tout scolastique authentique sera aussi un mystique en un certain sens, et tout mystique authentique un scolastique ».

\section{Husserl: «Étrange ; ainsi donc, depuis le sommet d'une montagne elle (Edith Stein)} contemple la clarté et l'ampleur de l'horizon dans sa transparence et sa légèreté merveilleuses, et pourtant, elle a en même temps l'autre tournure, la tournure vers l'intérieur, et la perspective de son ego». - AJ: «Oui, c'est possible en Dieu, mais seulement en Lui ». - Husserl : « En elle (Edith), tout est absolument authentique ; sinon il me faudrait dire que tout cela est apprêté et artificiel. Mais en définitive - les Juifs ont en eux le radicalisme et l'amour du martyre». Cette déclaration de Husserl fait référence à la vie d'Edith Stein comme carmélite et à sa mentalité.

[52] Un peu plus tard, il demanda: «Ne pourrai-je pas également venir une fois ou l'autre à Beuron?»- $\mathrm{AJ}$ : « Mais naturellement ». - Husserl : «Oh, mais je suis trop vieux. Je ne peux plus me convertir». - AJ : « Mais cela n'est pas du tout nécessaire. Personne n'attendra quoi que ce soit de semblable ou ne tentera de vous pousser à la conversion. Après tout, vous vous tenez dans la grâce, comme vous me l'avez dit une fois vous-même. C'est l'essentiel, et c'est pleinement suffisant ». Et de fait, il m'a posé un jour cette étrange question avec le plus grand sérieux (c'est quelque chose qui lui tenait vraiment à cœur) : « Sœur Adelgundis, ne suis-je pas moi aussi dans la grâce? »

\section{3 mai 1934. Visite de Husserl au couvent de Sainte Lioba (à Günterstal)}

C'était l'un de ses souhaits de me rendre visite avec sa femme afin de me remercier pour une lettre que je lui avais écrite pour son soixante-quinzième anniversaire. Il apporta des fleurs. Mais avant toute chose, il voulait en savoir plus sur l'entrée d'Edith Stein au Carmel de Cologne (fin avril 1934). Je lui fis la lecture du compte-rendu qui m'avait été envoyé par une participante. Husserl écouta avec grande attention. De temps à autre, il m'interrompait pour me poser des questions concernant les institutions et les usages de l'Église. Ce lui fut une véritable satisfaction qu'Edith Stein soit également estimée par l'Église et par l'ordre. Il me faisait l'effet d'un père se souciant de sa fille qui viendrait d'entrer par le mariage dans une nouvelle famille. Ce n'est pas sans fierté paternelle qu'il ajouta: «Je ne crois pas que l'Église compte dans ses rangs une néoscolastique de la qualité d'Edith Stein - Dieu merci, elle est autorisée à poursuivre son travail au couvent de Cologne ». 

Carmel de Cologne. Il déclara ingénument : «J'aurais pu être le père de la mariée. Quel dommage, sœur Adelgundis, que vous-même, au moins, n'ayez pas pu y assister!». Quand je lui répondis que je n'avais tout simplement pas d'argent pour le billet de train, il me rétorqua immédiatement : « Je vous l'aurais donné avec plaisir ». Je dus ensuite lui montrer des portraits de sainte Thérèse pour qu'il puisse voir l'habit qui est celui de l'ordre. Il conserva une petite reproduction, ainsi que le rite de notre prise de voile bénédictine et de notre profession de foi à Sainte Lioba. Puis il ajouta : «Je veux étudier cela de très près ".

Puis nous parlâmes de la situation présente, en particulier du désert spirituel de notre époque. Il s'interrompit soudain en disant: "Mais non. Comment parler de telles choses ici, dans le cloître? Ici, c'est un autre monde, un monde pour soi, hors de cette époque maligne. Ici, c'est comme au ciel ».

Puis il me fallut ouvrir pour lui les armoires de la bibliothèque. Il montra le plus grand intérêt, en particulier, pour les Pères de l'Église et sortit un volume d'Augustin. Il était manifestement ravi et intérieurement ému lorsqu'il visita la chapelle du Saint Sacrement et le chœur. Puis nous passâmes au jardin. Husserl était comme absorbé dans des pensées profondes; il nous a même laissées un moment avant de revenir un peu plus tard. Soudain, il prit ma main dans la sienne et me demanda avec une certaine urgence : «Pratiquez-vous également la direction de conscience?»- AJ : «Oui ». Husserl : «Oh, c'est une bonne chose. Je sais maintenant, sœur [53] Adelgundis, où me rendre quand le souci et la peine accablent mon âme. Je viendrai à Sainte Lioba et vous me réconforterez. Puis j'irai m'asseoir quelque part ici, dans le jardin, dans un coin tranquille, avec un Père de l'Église. Car je ne les connais pas encore du tout ».

Husserl : « Votre visite est une bonne façon d'achever l'année ». Il parla de la lettre de sœur Bénédicte (Edith Stein), qui lui avait écrit à propos de Duns Scot, et déclara : «Lui, est un mystique, il tient plus du mystique que saint Thomas d'Aquin. Aujourd'hui, l'Église et le clergé sont en train de sortir de la sécularisation et de la politisation et s'éveillent à l'intériorité authentique. L'Église a évité jusqu'à présent le «combat pour la culture $»^{5}$ parce qu'elle voulait conduire à son terme le processus de désécularisation afin de s'assurer de ses fidèles. Ce que les églises veulent, je le veux aussi : conduire l'humanité à l'aeternitas. Ma tâche consiste à le tenter par le biais de la philosophie. Tout ce que j'ai écrit jusqu'à présent n'est que travail préparatoire ; c'est seulement la mise en place de méthodes. Malheureusement, le cours d'une vie est loin de suffire pour parvenir au cœur, à ce qui est essentiel. Il est si important que la philosophie soit extraite du libéralisme et du rationalisme et conduite de nouveau à ce qui est essentiel, à la vérité. La question portant sur l'être ultime, sur la vérité, doit être l'objet de toute vraie philosophie. C'est le travail de ma vie.

«Je demeurerai un hérétique. Si j'avais quarante ans de moins, je me laisserais volontiers conduire par vous jusqu'à l'Église. Mais voyez-vous, je suis si vieux à présent, et comme j'ai toujours fait les choses de façon si méticuleuse, j'aurais besoin d'au moins cinq ans pour chaque dogme. Vous pouvez vous imaginer quel âge il me faudrait atteindre pour parvenir à la fin. Me conserverez-vous malgré tout votre amitié ? 
«Comme vous le voyez, j'ai toujours différentes éditions du Nouveau Testament sur mon bureau et à ma table de travail. Et lorsque j'ai été très malade il y a quelques années, sœur Bénédicte s'asseyait auprès de mon lit et m'en lisait des passages ».

- AJ : "Oui, et vous pouvez aussi faire appel à moi si jamais vous étiez de nouveau malade, ou si c'était votre dernière maladie ». - Husserl : «Oh, j'imagine fort bien que vous serez là quand je serai mourant, et que vous me lirez le Nouveau Testament au moment où je passerai dans l'éternité ».

\section{8 avril 1935}

Visite pour féliciter Husserl à l'occasion de son soixante-seizième anniversaire. Il en fut si touché qu'il eut les larmes aux yeux et put à peine parler. À cette époque, de nombreux amis et étudiants s'étaient déjà détournés de lui - un effet des positions antisémites du troisième Reich. Il manifestait un intérêt enthousiaste à propos de mes vœux dont la profession était prévue le $1^{\mathrm{er}}$ mai. Et quand je l'eus solennellement invité, il me dit : « Il y a huit jours, vous ne m'auriez pas vu si heureux. Car j'étais très inquiet de la question de savoir si oui ou non les conférences que je dois donner sous peu à Vienne et à Prague ${ }^{6}$ [54] me permettraient d'être présent pour cette occasion festive, et il est très important pour moi d'y être. Non, j'aurais reporté ces conférences; je dois assister à cette cérémonie quoi qu'il en coûte. Mais à présent tout est réglé. Je ne partirai pas avant l'automne ». Il me demanda de lui expliquer le rite de la profession. Pour accéder à son souhait, je lui en laissai une copie qu'il souhaitait pouvoir garder pour pouvoir l'étudier de près.

\section{$1^{\text {er }}$ mai 1935}

Husserl et sa femme arrivèrent à temps pour la cérémonie de ma prise de vœux perpétuels à Sainte Lioba. Depuis les sièges privilégiés qu'ils occupaient, ils purent tout voir et, comme je l'appris plus tard, ils suivirent la sainte célébration avec le plus grand intérêt et le plus profond recueillement. À l'issue de la cérémonie qui dura à peu près deux heures et demie, je fus appelée à la bibliothèque où ils me saluèrent et me félicitèrent - touchés et émus l'un et l'autre, Husserl jusqu'aux larmes. Il m'offrit un grand portrait photographique de lui, orné de sa signature, et sa femme une magnifique calla en fleurs. Soudain, il porta la main à son cœur, fut pris de vertige, et nous avons eu beaucoup de mal à le rétablir grâce à un verre de vin. Il ne put que murmurer, avec un doux sourire : «j'étais submergé de joie ; c'était trop beau ».

\section{4 septembre 1935}

Husserl : « L'honnêteté de l'intellect est à la philosophie ce que la probité du cœur est à la religion. Toute ma vie j'ai lutté, je me suis même battu, pour cette honnêteté et là où les autres étaient satisfaits depuis longtemps je n'ai cessé de continuer à me questionner et d'examiner si même un semblant de malhonnêteté ne persistait pas à l'arrière-plan. Tout mon travail, même aujourd'hui, ne consiste qu'à examiner et à inspecter encore et encore, car tout ce que j'avance n'est, après tout, que relatif. On doit avoir le courage d'admettre et de déclarer que quelque chose qu'on considérait encore vrai la veille mais dont on voit aujourd'hui que c'est une erreur, est bel et bien une erreur. Rien n'est absolu ici. Il m'est arrivé de dire cela, il y a de nombreuses années, à mon étudiant le père franciscain $\mathrm{P}$. Il était très brillant et, philosophiquement, il suivait mes développements, quoique seulement jusqu'à un certain point. Mais il ne parvenait pas à trouver le courage de revenir en arrière et de 
reconnaître une erreur. La seule chose qui comptait pour lui, même en philosophie, c'était l'absolu. C'est là que nos chemins divergent.

"Je regrette profondément (ici Husserl devint très sérieux et insistant, presque agité) d'être si mal compris. Personne ne m'a suivi depuis le grand bouleversement qui a eu lieu dans ma philosophie, depuis mon tournant intérieur. Quand les Recherches logiques ont paru en 1901, ce n'était que l'œuvre tout à fait modeste d'un débutant - et aujourd'hui, on juge Husserl à partir de ces Recherches logiques. Mais pendant de nombreuses années après leur parution, je ne savais pas bien où tout cela me menait. Je n'étais pas au clair sur moi-même, et pour mon malheur, tout le monde est resté bloqué sur ce livre. Mais ce n'était là qu'un [55] chemin, fût-il un chemin nécessaire. Même Edith Stein ne m'a suivi que jusqu'en 1917... On va jusqu'à affirmer que j'en serais revenu à Kant. Voilà à quel point je ne suis pas compris ! Comme on a remarqué que ma phénoménologie était la seule philosophie ayant également des liens avec la scolastique, comme beaucoup de théologiens ont étudié mes Recherches logiques - mais malheureusement pas les ouvrages ultérieurs - on a prétendu que j'avais commencé par embrasser la religion avant de rechuter dans l'incroyance!

«En dépit de tout, il m'est arrivé de croire - et aujourd'hui c'est plus qu'une croyance, aujourd'hui c'est un savoir - que c'est précisément ma phénoménologie, et elle seule, qui est la philosophie dont l'Église puisse faire usage, car elle converge avec le thomisme et étend la philosophie thomiste. Pourquoi l'Église tient-elle au thomisme avec tant de rigidité? Si l'Église est vivante, alors elle doit poursuivre son développement aussi dans la phénoménologie. La Parole de Dieu est toujours la même : elle est éternelle. Mais son interprétation philosophique est dépendante de personnes particulières, vivant à une certaine époque. Elle est donc relative. Songez-y: Thomas était précédé par Aristote, le grand païen ; il a construit sur sa base. Thomas était luimême un esprit particulièrement brillant et productif, et c'est de façon autonome qu'il a poursuivi son travail. Mais rien n'est aussi stérile que le néo-thomisme (Husserl n'avait en particulier rien de bon à dire des néo-thomistes français). Il n'a que Thomas pour base et c'est pourquoi il finit par se pétrifier. Il faudra bien un jour que la philosophie catholique croisse au-delà de tout cela.

47 «J'ai une tâche, une mission qui m'a été confiée par Dieu. Je dois l'accomplir - c'est ce pourquoi je vis. Cela fait trente-cinq ans maintenant que je continue quotidiennement et sans interruption à travailler, y compris à de nouvelles choses. Je n'ai pas eu le temps de préparer mes manuscrits pour une publication. À part Fink ${ }^{7}$, je n'ai pas eu un seul étudiant depuis quatre ans à qui m'ouvrir. Il m'était alors impossible d'exprimer le fond de ma pensée. J'en ai souffert, mais il n'y avait rien à faire. Et maintenant qu'à soixantedix ans - soixante-seize à présent - je n'ai pas d'étudiant ni la possibilité d'enseigner, il n'y a pas d'école qui soit susceptible de prolonger mes pensées et de les publier. Le prophète est la bouche de Dieu. Très directement. Il n'enseigne pas; il ne travaille pas. Il n'a pas de tâche au sens authentique et ordinaire ».

Je répondis : «Et pourtant le prophète est aussi un humain, traversé par la mission qu'il a reçue de Dieu. Il ressent dans l'effroi sa faiblesse humaine (Jérémie) et se trouve purifié (Isaïe); car en lui la nature aussi est à l'œuvre, pas seulement la vocation et la grâce. Il peut accepter sa tâche ou bien se démettre. Vous êtes un prophète, monsieur le Professeur, car vous avez quelque chose à dire à notre époque. Vous avez une mission pour l'humanité !» 

servir les deux confessions chrétiennes. Peut-être se rendra-t-on compte un jour que parvenu à un certain point, j'ai dû refondre ma pensée et qu'ainsi je suis resté fidèle à moi-même.

«Il y deux groupes parmi mes étudiants - les uns sont les boucs, [56] les autres les agneaux. En ce qui concerne la confession ou la religion, les boucs sont ceux qui veulent me voir tels qu'ils sont eux-mêmes - religieux et confessionnels -, mais non pas fondamentalement libre, délié, objectif, franc et honnête. Les agneaux - et je m'entends assez bien avec eux, qu'ils soient strictement catholiques ou protestants - sont ceux qui m'accordent le droit, eu égard à la religion, d'avoir une personnalité libre, ceux qui me prennent et me considèrent tel que je suis. C'est pourquoi j'ai toujours et depuis si longtemps été dans les meilleurs termes avec eux. Dans les nombreuses années qui se sont écoulées depuis 1916, il n'y a jamais eu la moindre faille dans notre amitié, sœur Adelgundis, parce que vous n'avez jamais cherché à m'attaquer sur la question religieuse ".

dernier Goethe: "Quelle merveilleuse manière ils avaient, à l'époque, de parler des choses, de façon à la fois profonde et intelligente, et pourtant ni trop profonde ni trop scientifique. C'était une belle manière de philosopher, mais ce n'était pas de la philosophie au sens rigoureux du terme. Finalement, c'était de l'expressionisme au meilleur sens du terme. Aujourd'hui, plus personne n'a de manière ni de méthode spirituelle ; tout est bâti sur des sentiments, sur des impulsions et des impressions, sans le moindre fondement en raison ». - AJ : « Pascal ménage une place pour quelque chose qui se situe entre le sentiment et la ratio et qu'il nomme logique $d u$ cœur* ${ }^{*}$. Husserl : «Oui, c'est excellent de considérer et de juger les choses non pas avec une raison froide mais d'une façon calme et claire, avec le cœur (ce qui, du reste, ne relève pas du sentiment) ».

Chez les Husserl pendant une heure. Ils parlèrent avec beaucoup d'animation et de plaisir de l'hospitalité et de la grande compréhension dont ils avaient été l'objet à Prague à l'occasion des conférences ${ }^{8}$. Puis on me laissa seule avec le maître. J'étais sur le point de partir quand il commença à philosopher et - tandis que nous étions débout se mit à parler de sa philosophie pendant au moins trois quarts d'heure. Il acheva en disant sobrement: «La vie humaine n'est rien d'autre qu'un chemin vers Dieu. J'essaie d'atteindre ce but sans preuve ni méthode ni soutien théologiques, c'est-à-dire de parvenir à Dieu sans Dieu. Je dois pour ainsi dire éliminer Dieu de mon existence scientifique afin de tracer un chemin qui mène à Dieu pour les gens qui, contrairement à vous, ne reçoivent pas de l'Église la certitude de la foi. Je sais que cette façon de procéder pourrait être dangereuse pour moi si je n'étais pas moi-même un homme profondément lié à Dieu et croyant au Christ ». pleurésie qui fut loin d'être bénigne. Il mit du temps à s'en remettre. Comme je fus tout l'hiver en déplacement pour des conférences, ce n'est qu'à l'occasion de son soixantedix-septième anniversaire, le 8 avril 1936, que je le revis. 

qu'il s'écria - et avant même que j'aie pu [57] lui parler - avec une ferveur presque juvénile, comme il arrive qu'une joie anticipée la produise: "Vous devez nous accompagner à Rapallo; nous partons dans huit jours; le médecin a stipulé que je devais partir pour le Sud pour ma convalescence. Demandez à votre prieure la permission de faire le voyage. J'ai besoin d'une infirmière et d'une étudiante à qui je puisse m'ouvrir. Ce sera un moment merveilleux et je me réjouis d'avance infiniment de ces semaines. Mais vous devez venir ». Le 15 avril 1936, je fis le voyage à Milan par le Saint Gothard en compagnie d'Elli [Elisabeth], la fille de Husserl. Nous arrivâmes à Rapallo dans la soirée du 17. 
$\mathrm{Au}$ dîner, conversation à propos de l'Inquisition, des ordres religieux, d'Ignace de Loyola. Débat autour de l'ouvrage de Hugo Ball. Husserl, dont l'idéal de vie est la sage modération des Stoïciens, rejette de la façon la plus vive la brusquerie immodérée et la rigueur ardente, sans compromis, des martyrs byzantins.

Au cours du dîner, où, pour notre plus grand plaisir, il mangea avec appétit, Husserl fut très animé. L'achat de lunettes sombres avait probablement changé ses dispositions physiques et mentales. Il évoqua de façon détaillée son maître Franz Brentano. Bien qu'il se fût effectivement marié alors qu'il était prêtre, il n'en est pas moins resté si profondément prêtre et catholique en son for intérieur, qu'il s'en est un jour violemment pris à Husserl alors que ce dernier avait soutenu que le culte des saints était de l'idolâtrie. C'est avec l'aval de Brentano que Husserl a donné une série de cours sur les preuves de Lotze en faveur de l'existence de Dieu. À l'exception de lui-même, Aristote et Thomas étaient les seuls philosophes dont Brentano reconnaissait la validité.

Husserl ne cessait de répéter qu'il portait Guardini en haute estime. Il affirma aujourd'hui que dans son livre Spiegel und Gleichnis ${ }^{13}$, que je lui avais offert, il s'accordait mot pour mot avec Hedwig Conrad-Martius. De nouveau, il rejeta Theodor Haecker en lui reprochant son manque de créativité ; quoi qu'il en soit, il lui porte moins d'intérêt qu'à Guardini.

Dans la soirée, nous échangeâmes encore longtemps à propos du symbolisme et de l'exégèse historique et génétique de la Bible. Puis Husserl s'étendit assez longuement (jusqu'à 23h) sur des aspects de ce qu'il nommait l'eschatologie statique de notre Terre : «Séparée de Dieu (l'infini), la finitude aspire à retourner à l'infini. La grâce est la liberté de Dieu ». Husserl défendit la conception selon laquelle ce n'est qu'en un combat constant que l'homme s'approche de son Dieu, de l'infini. En pratique, cela se produit sans le concours de la grâce. Si donc l'homme a assurément besoin de Dieu, Dieu a également besoin du monde et des humains. Je l'ai contredit sur ce point. Sans solution ni accord, nous nous séparâmes tard ce soir-là - quelque peu chagrinés et attristés de n'être pas parvenus à nous entendre.

\section{Les dernières années d'Edmund Husserl (1936-1938)}

\section{[129] Été 1936}

Après leur retour de Rapallo, les Husserl restèrent tout l'été et jusque tard dans l'automne à Kappel, près de Neustadt (en Forêt-Noire). L'atmosphère y était très apaisée et remplie d'une grande bienveillance envers les Husserl. Avec la délicatesse de sa sensibilité, Husserl a réagi très vivement à toute l'hostilité à l'égard des Juifs qui se développait à cette époque. Cet été là, il travailla très intensément au commencement d'un nouvel ouvrage dont les prolégomènes avaient récemment paru à Belgrade ${ }^{14}$ (un éditeur allemand n'avait plus le droit d'accepter des ouvrages de Husserl). Son état était variable. L'isolement rural du village d'altitude, le vaste paysage de la Forêt-Noire qui, ici particulièrement, prend l'apparence d'un plateau, avec ses larges panoramas et la chaîne des Alpes qui brille à l'horizon les jours de foehn - tout cela fit du bien à son âme qui devenait très émotive. Je crois que le paysage morave de sa jeunesse se dressait ainsi devant lui. Il ne cessait de revenir sur sa parenté généalogique avec Adalbert Stifter et Rainer Maria Rilke. L'air frais et fort de la montagne (Kappel se situe entre 900 
et 1000 mètres d'altitude) stimula sa productivité. Parfois, une véritable angoisse semblait le saisir à l'idée qu'il pourrait n'être plus capable d'achever son ouvrage. Puis, il se remettait au travail, fiévreusement et sans répit, dans sa chambre aux fenêtres fermées, et il était impossible d'obtenir qu'il sortît pour se promener. Mme Husserl m'invita à leur rendre visite quelques jours afin de lui permettre de se détendre brièvement. Et de fait, il fut quelque peu distrait par ma présence et contraint de sortir marcher avec moi ; à cette occasion, il rompit son profond silence et recommença à parler. Mais lors des repas, en revanche, il fut la plupart du temps spirituellement distant et bien plus silencieux que d'habitude ; il semblait, d'un côté, oppressé par des pensées et des préoccupations bien lourdes, mais, d'un autre, profondément ému par des images intérieures.

73 Je me souviens de deux longues promenades que nous fîmes tous les deux. Il parla longuement de son Allemagne bien-aimée ainsi que de la haine imméritée qui s'est abattue sur les Juifs allemands comme un horrible flot d'ordures. Cela faisait profondément souffrir Husserl car il était allemand du fond de son être. Tout cela lui était inconcevable. Nous étions ensemble au cimetière de Kappel, et il me raconta qu'il avait lui-même choisi une tombe ici comme lieu de son dernier repos. Il espérait pouvoir dormir ici en paix jusqu'à la résur-rection. Pendant la promenade, il dit :

[130] « Ces derniers jours, j'ai reçu d'Amérique un journal dans lequel un Jésuite - donc l'un des vôtres, sœur Adelgundis - m'a présenté comme un philosophe chrétien. Je suis consterné par cette initiative précipitée et excessivement zélée, dont je ne savais rien. Comment peut-on faire une chose pareille sans me poser la question ?! Je ne suis pas un philosophe chrétien. S'il vous plaît, veillez à ce qu'après ma mort on ne me fasse pas passer pour tel. Je vous ai déjà souvent dit que ma philosophie, la phénoménologie, est censée n'être rien d'autre qu'un chemin, une méthode, destinée à montrer justement à ceux qui se sont éloignés du christianisme et des églises chrétiennes le chemin qui reconduit à Dieu ».

\section{3 mars 1937}

Husserl : "Comment le christianisme, considéré comme science, contribue-t-il à la fondation? Il porte en lui l'évidence - non pas, bien sûr, une évidence absolue toujours et partout. Mais nous devons également nous efforcer de reconnaître les évidences relatives. Sinon, nous dissolvons la vie; sinon, nous détruisons la vie chrétienne, qui porte pourtant en elle-même l'évidence en faveur de sa crédibilité. Il ne fait aucun doute que nous pouvons également approcher le christianisme par les moyens de la science, et c'est précisément ce qu'ont fait la scolastique, le droit canon et l'administration ecclésiale - mais plus important que tout cela, il y a la vie vivante et dans ce domaine, l'évidence relative est acceptée sans difficulté. Y a-t-il, dans la vie religieuse, évidence plus assurée et plus authentique que la prière ? Naturellement pas le babillage ! Et pourtant, la prière ne relève pas de l'évidence ultime, absolue. Il en va de même pour la science. La source de toutes ses erreurs dans les trois derniers siècles - et même la scolastique n'y échappe pas - fut précisément qu'en raison d'un fort scepticisme, elle a perdu le sol du seul étant véritable ».

\section{8 avril 1937}

Entretemps, Husserl s'est trouvé tout à fait isolé. Car le national-socialisme avait veillé à ce que le cercle de ses amis se rétrécisse toujours davantage et que la science officielle 
prenne elle aussi ses distances avec lui. Lorsque je suis venue lui souhaiter son soixante-dix-huitième anniversaire, il était seul. Nous avons bien discuté :

«Lorsque le christianisme, c'est-à-dire l'Église, a adopté la philosophie grecque (Aristote), il s'est jeté dans un conflit désastreux, impossible à résoudre. Car le résultat fut que la philosophia perennis et revelata [la philosophie éternelle et révélée] ${ }^{15}$ et la philosophie autonome en vinrent à s'opposer l'une à l'autre. Lorsque le bon Dieu créa le monde, il a également créé la philosophie (un doux sourire plein d'humour accompagna ces mots), et elle n'était certainement pas mauvaise, mais bonne. Lorsqu'on pratique la recherche et qu'on pense dans la foi et de façon religieuse - et tout philosophe est religieux - lorsqu'on n'accepte pas sans plus la vérité révélée mais qu'on en fait également l'objet de son enquête, oui, lorsqu'on s'expose soi-même à l'“enfer d'une skepsis totale" dans le but de fonder la vérité de façon téléologique - alors les scolastiques [131] refusent de suivre et seuls les néo-scolastiques sentent qu'il leur faut aller au-delà de Thomas (il est certain que Thomas, c'était quelque chose de très impressionnant, un phénomène colossal).

«Mais les néo-scolastiques ont peur de se détourner de toute révélation, des dogmes, de Dieu lui-même - et même si c'est seulement en pensée (de façon hypothétique). Dans ma réduction phénoménologique, je souhaite seulement rassembler toutes les philosophies et les religions au moyen d'une méthode de connaissance universellement valide. L'ontologisme est une doctrine fallacieuse et extrêmement dangereuse. Les néoscolastiques étaient d'accord avec moi à l'époque où j'en étais là. Mais plus tard, ils n'ont pas compris que c'était seulement une station sur mon chemin. On abstrait l'être pur et simple et l'on met hors-jeu la conscience dans laquelle seule, pourtant, l'être devient et demeure vivant. La matière elle-même est quelque chose de spirituel ; c'est seulement qu'elle se tient au degré le plus bas de l'ordre spirituel ».

81 Durant l'été, les Husserl ont été contraints, pour des " raisons raciales », de quitter leur cher appartement au deuxième étage du 40 Lorettostrasse, où ils vécurent heureux de nombreuses années et où le grand et magnifique bureau du maître nous semblait une sorte de temple, lié précisément à ce lieu. Ils y ont vécu exactement vingt ans. Auparavant, ils avaient vécu dans Bayernstrasse. L'arrière des deux immeubles se font face. C'est là que j'ai rencontré Husserl pour la première fois, en 1916. Les choses se sont passées ainsi : un jour, je reçus une carte postale manuscrite de sa part m'invitant à lui rendre visite dans son appartement pour récupérer quelques ouvrages philosophiques qu'une de ses étudiantes m'avait légués dans son testament. Dans la grande bibliothèque de son étudiante décédée, Husserl avait lui-même choisi les livres pour moi qui étais une étudiante de premier semestre : l'Introduction à la philosophie de Windelband, l'Esquisse de Paulsen ${ }^{16}$ et une anthologie (Reclam).

Le déménagement de l'été 1937 ne fut pas une mauvaise chose et j'ai souvent eu l'occasion d'admirer la sagesse de la Providence qui a accordé au maître, pour la dernière station de son chemin terrestre, une si jolie maison, à mi-pente, au 6 Schöneckstrasse (la maison Faist). La maison ressemblait presque à une rotonde; où qu'on se tînt, on pouvait jouir des plus belles vues de chaque côté. Depuis la rue, on franchit une petite passerelle, au niveau du sol et qui conduit à l'appartement, lequel était fait d'un petit nombre de très grandes pièces. Une terrasse spacieuse, s'étendant sans interruption des trois côtés de la maison, relie les pièces les unes aux autres. D'ici, on peut profiter d'une vue indescriptible sur la ville tout entière jusqu'au Kaiserstuhl et aux Vosges ; on devine même le lit du Rhin entre les montagnes. 

semaines environ à Breitnau, près de Hinterzarten (en Forêt-Noire). Il appréciait ce lieu perché à 1000 mètres d'altitude pour son isolement et son caractère. [132] La propriétaire de l'auberge "Zum Kreuz », une femme qui appréciait les Husserl depuis de nombreuses années, n'a pas hésité à l'accueillir de nouveau, même en 1937. Mais elle dut le faire en secret et Husserl prenait ses repas seul, dans une pièce adjacente. Je lui ai rendu visite par une magnifique journée d'été. Avec une joie sereine, il vint me chercher à la descente du bus et nous passâmes la journée ensemble, jusqu'au soir. La plupart du temps, nous étions dehors. La vue des prairies en fleurs et des champs pleins de fruits le remplissait d'une joie mélancolique. L'époque et tout ce qui se passait en Allemagne l'oppressaient énormément. Il s'attachait d'autant plus fortement à la loyauté et aux sentiments du petit nombre des vieux amis qu'il avait encore. De façon bien compréhensible, il craignait les personnes qu'il ne connaissait pas. À la mijournée, nous avons visité l'église de Breitnau, qui se trouve au milieu du cimetière, entourée d'un muret blanc. Nous avons remonté et descendu les allées entre les tombes, en faisant souvent des pauses. Husserl regardait au loin, pensif ; son âme était assombrie par la tristesse.

84 Avec calme mais non sans peine, il me raconta qu'il avait été invité en France pour présider le congrès Descartes ${ }^{17}$, mais que le ministre de la culture, [Bernhard] Rust, lui avait refusé la permission de voyager, pour la raison que Husserl n'était pas en mesure de représenter la philosophie allemande à l'étranger. C'est pourquoi Rust avait suggéré pour cette tâche le professeur [Ernst] Krieck, de Heidelberg. Du reste, Krieck venait tout juste de publier sur Husserl un article affligeant, et même lamentable, pour la nouvelle Brockhaus ${ }^{18}$, ce que nous avons tous trouvé extrêmement blessant, pour ne pas dire abject. Bien que nous ayons tenté de le cacher à Husserl, il a fini par l'apprendre un jour de quelqu'un d'autre. Il souffrait profondément de la façon dont l'Allemagne le traitait à présent, après qu'il lui avait consacré toute son énergie et tout son travail. Même le fait que le poste de président du congrès Descartes demeura vacant (puisque la France a naturellement rejeté la proposition du professeur Krieck) ne put venir à bout de son humeur maussade. « Voyez-vous, sœur Adelgundis, mes cendres elles-mêmes ne seront pas dignes de reposer dans la terre allemande ». Et, tout en jetant vers les tombes un regard qui, pour la seule et unique fois, n'était pas tout à fait dépourvu d'amertume : « Même ici, il m'est impossible de trouver la paix. Vous voyez à quel point l'Allemagne en est déjà arrivée! Peut-être que même ici, dans ce cimetière de village, des fanatiques profaneraient ma tombe s'ils venaient à la trouver ».

Husserl fut très fatigué cet après-midi-là et nous avons dîné dans le jardin de la petite ferme où il séjournait. Ce jour-là, je ne parvins pas à rehausser son humeur. Nous avons évoqué Stifter, son compatriote, qu'il aimait profondément et dont il partageait tellement la dignité noble et tranquille. Durant la dernière année de sa vie, il relut Stifter avec plaisir et il parlait volontiers de ce qu'il avait lu. Il se trouve que Witiko fut le dernier livre que Husserl ait lu ${ }^{19}$.

Husserl n'aura pas eu l'occasion de profiter longtemps des conditions de travail favorables offertes par sa nouvelle maison. Le 10 août 1937, les Husserl fêtèrent [133] en toute discrétion leur cinquantième anniversaire de mariage. C'était leur vœu exprès que personne ne le sache, pas même dans le cercle de leurs amis proches. Tôt le matin, alors qu'il s'habillait dans sa salle de bain, Husserl glissa et, en tombant, se fit manifestement une blessure interne. Les médecins estiment que c'est cette chute qui 
sema les germes de la maladie qui devait l'emporter. Il contracta une pleurésie exsudative qui, bien qu'elle ait été parfois interrompue par les brèves améliorations d'une santé chancelante, ne cessa de l'épuiser. Elle devint une maladie inhabituellement longue, douloureuse et pénible, qui dura huit mois.

Le docteur Otto, le vieux médecin de famille qui le traitait, déclara qu'il n'avait jamais rencontré, dans ses nombreuses années de pratique, un cas tel que celui-ci. D'un point de vue médical, le patient n'aurait pas dû pouvoir vivre, c'est-à-dire exister, jusqu'en avril 1938. Il s'alimenta de moins en moins. La fièvre variait mais ne cessait de consumer les forces et la substance de son corps. Deux exsudats sévères se formèrent, qu'il fallut ponctionner. Puisque, à la demande de Husserl, je prenais parfois en charge ses soins infirmiers, j'ai également aidé à la réalisation d'une ponction: il y avait quelque chose de bouleversant dans la souffrance silencieuse du patient. Pour le reste également, Husserl se plaignait fort peu et se satisfaisait de tout, à l'exception du fait qu'il était presque impossible de le faire manger et que toute tentative pour l'en persuader l'agaçait. Son corps fondit mais son esprit n'en poursuivit pas moins, incontesté, la vie rigoureuse qui était la sienne. On avait parfois l'impression que seul son esprit était encore là. Sa vie était réellement une énigme pour les médecins. Quoi qu'il en soit, elle défiait toute expertise médicale. L'esprit ne cessait d'être actif et dispensait sa richesse.

89 À mon arrivée, Husserl était levé. Parfois, lorsqu'il se sentait bien, il se levait dans la soirée. Nous avons dîné l'un à côté de l'autre dans son bureau. Il tenait ma main dans la sienne pendant la conversation. Dehors, c'était l'une de ces rares belles journées de début d'automne qui s'achevait. Tout était tranquille. Le soleil descendait lentement derrière les Vosges. Magnifique et solennelle, la silhouette de la cathédrale se dressait dans la lumière dorée du soir, au-dessus du labyrinthe gris et plusieurs fois centenaire des toits de la ville, cette ville qui lui était si chère. La lumière du soir brillait dans ses yeux. Ils étaient complètement absorbés dans la vue de la chaîne des montagnes qui luisait doucement à l'horizon et de la ville à ses pieds. C'est alors qu'il rompit le silence profond qui s'était prolongé longtemps entre nous. Sans détourner le regard de la cathédrale, il dit de façon douce et pénétrante :

90 "Je n'imaginais pas que mourir fût si difficile. Je me suis efforcé, ma vie durant, de me débarrasser de toute vanité, et après avoir tracé mon propre chemin, maintenant que je suis si complètement conscient de la responsabilité de ma tâche, maintenant que dans mes conférences récentes à Vienne [134] et à Prague ${ }^{20}$, puis dans mon dernier écrit, $L a$ crise des sciences européennes et la phénoménologie transcendantale (Belgrade, 1936), je suis sorti de ma coquille pour la première fois, de façon complètement spontanée, et suis parvenu à accomplir un petit commencement, maintenant il faut que je m'interrompe et que je laisse ma tâche inachevée. Maintenant même, alors que je suis fini, je sais que je commence à nouveau, parce qu'être fini signifie recommencer.

91 «Et j'imaginais que ce serait si magnifique quand j'aurais accompli ma tâche, ma tâche pour le monde, et que par la phénoménologie j'aurais montré aux hommes une nouvelle manière d'être responsable afin de les libérer de leur vanité et de leur ego. Mon Dieu! depuis ma jeunesse, je me suis réellement battu contre les vanités et, parvenu en ce point, je m'en serais débarrassé presque complètement, y compris de la vanité professionnelle sans laquelle une personne jeune ne peut pas travailler : être honoré et admiré par mes étudiants. Oui, maintenant, quelques minutes avant de 
mourir, je me serais presque consacré complètement au Nouveau Testament et je n'aurais lu que ce livre. Quel beau crépuscule de vie ç'eût été ! Et après avoir achevé ma tâche impérative, voici quel aurait finalement été mon sentiment : maintenant je puis faire ce par quoi je me connais moi-même. Personne ne peut se connaitre vraiment soimême sans lire la Bible.

"Je considère que votre tâche, ma chère enfant, consiste en particulier - ô, puissiezvous vous y tenir ! - à gagner dans l'amour les âmes des jeunes gens à l'amour, et à les protéger des grands dangers qui menacent l'Église: de la vanité stérile et du formalisme rigide. Promettez-moi de ne jamais dire quelque chose seulement parce que d'autres l'ont dit. Les grandes, les saintes prières de l'Église sont en danger constant d'être vidées de leur contenu faute d'être alimentées par une vie personnelle. L'Église rejettera mon œuvre - peut-être pas la jeunesse dans l'Église, vos amis - parce qu'elle voit en moi le plus grand ennemi de la scolastique, ou du moins de la néo-scolastique ». Puis avec un sourire tranquille et ironique, il ajouta : «Oui, Thomas, je le révère - mais il n'était pas non plus un néo-scolastique ».

Pendant les mois d'hiver, il diminua visiblement. En mars, son état devint si sérieux que je passai souvent les nuits à veiller à son chevet. Il dormait beaucoup et demeurait dans un demi-sommeil, sans qu'on pût jamais dire qu'il eût totalement perdu connaissance. Souvent, il semblait pris dans des conversations avec lui-même ou bien avec un interlocuteur invisible. Le plus souvent, quand j'arrivais en début de soirée, il était dans un demi-sommeil, et je demeurais assise en silence, sans mouvement, à côté de son lit jusqu'à ce qu'il se réveille. Alors à chaque fois, une grande joie se peignait sur ses traits, qui devenaient de jour en jour plus transfigurés et spirituels. Ses lèvres formaient toujours un mot de remerciement, d'amitié. Il aspirait à présent à exprimer cela ouvertement, tandis qu'à l'époque où il était en meilleure santé, il ne le faisait que rarement et seulement dans des occasions exceptionnelles.

[135] Je me souviens que durant ces jours, puisqu'il me parlait parfois de sa jeunesse, il m'a rappelé la devise de l'orphelinat franconien à Halle. Il le fit peut-être en référence aux premières et difficiles années de sa carrière scientifique, puisque cette devise devant laquelle il est si souvent passé avait tant signifié pour lui : «Les adolescents se fatiguent et s'épuisent, les jeunes ne font que chanceler, mais ceux qui espèrent en Yahvé renouvellent leur force, ils déploient leurs ailes comme des aigles, ils courent sans s'épuiser, ils marchent sans se fatiguer » (Is, 40, 30 sqq.).

Pendant ma garde de nuit, nous avons eu la conversation suivante, que je retranscrivis immédiatement la nuit même. Il me sembla qu'il obéissait à des lois qui relevaient déjà d'un autre monde. Sans introduction ni référence, il commença soudainement : « Avant tout commencement, il y a toujours l'ego, qui est, pense et cherche des relations dans le passé, le présent et le futur. Mais tel est précisément le problème difficile : qu'y avait-il avant le commencement?»

dernières conversations n'étaient plus celles du phénoménologue qui se sentait appelé à accomplir une tâche pour le monde, mais celles du professeur aimé et de l'ami qui s'en allait et qui paraitrait bientôt devant la face de Dieu. Comme je souhaitais voir son âme délivrée de tout trouble et détournée de toute contingence extérieure! Je répondis donc: "Avant le commencement, il y a Dieu - comme le dit Jean: "Au 
commencement était le Verbe et le Verbe était avec Dieu et le Verbe était Dieu" ». Husserl : «Oui, tel est justement le problème qu'on ne peut résoudre que petit à petit ».

Après un moment, il continua pensivement: "Dans l'étude de la philosophie, les Présocratiques sont infiniment importants. Veillez donc à ce qu'ils soient lus. Aristote dit : on commence avec l'étant; la séparation de l'épistèmè et de la doxa advient avec la découverte créative de l'étant. - La philosophie est la volonté passionnée d'atteindre la connaissance de l'étant. Ce que j'ai écrit dans mon livre est très difficile. Toute philosophie est philosophie du commencement, philosophie de la vie et de la mort. Toujours et encore, nous recommençons depuis le début, et nous le faisons de plus en plus. Ma philosophie s'est toujours efforcée de s'éloigner de la subjectivité en direction de l'étant ». Environ une heure après, comme absorbé dans sa pensée, il dit: "Tout bien réfléchi, c'est toujours l'ego que nous posons, non pas une chose, un arbre ou une maison ».

Puis il s'assoupit de nouveau. Tandis que je repensais à ses paroles, il me revint que dans l'une de nos récentes conversations, nous avions parlé de ses leçons philosophiques. Je lui avais alors posé la question qui m'avait si souvent taraudée quand j'étais étudiante : «Pourquoi n'avez-vous jamais parlé de Dieu avec nous? Vous savez, je L'avais perdu à l'époque et je Le cherchais dans la philosophie. De leçon en leçon, j'attendais de Le trouver par le moyen de votre philosophie». Il m'avait répondu : «Ma pauvre enfant, comme je vous ai déçue, et quelle responsabilité [136] je dois endosser de n'avoir pu vous donner ce que vous cherchiez. Je n'ai jamais présenté quoi que ce soit d'achevé dans mes leçons; je n'ai jamais philosophé que sur ce qui me mettait en mouvement. À présent, je serais finalement allé assez loin pour tenir des leçons qui pourraient vraiment offrir quelque chose aux jeunes gens, mais c'est trop tard maintenant ».

\section{4 avril 1938, Jeudi Saint}

Durant les deux dernières semaines, il a fallu qu'une infirmière s'installe en service constant. Kläre Immisch, une infirmière de la Croix-Rouge, m'a rapporté la conversation suivante, qui se rattache à une lettre de la fille de Husserl, Elli Rosenberg, qui était arrivée d'Amérique. À deux heures et demie de l'après-midi, il dit : «Papa a commencé son centième semestre de travail (ce qui correspond de fait à peu près à l'ampleur de la carrière d'enseignement académique de Husserl), et on lui a accordé de nouvelles promotions. Une nouvelle période de travail productif commence, qui promet des connaissances essentiellement nouvelles pour les deux prochaines années. Bien entendu, le travail productif s'approchera ensuite graduellement de sa fin. Chacun de ceux qui ont vécu ces dernières années avec moi dans un esprit de continuité saura que ce qui s'est joué en moi n'était pas qu'un événement vain. Cela aura et aura eu lieu : la vie et la mort, l'aspiration ultime de ma philosophie. J'ai vécu en philosophe et je veux essayer de mourir en philosophe. Ce qu'il m'a été donné de créer et ce qui peut m'être encore accordé se trouve entre les mains de Dieu ».

Tout cela fut prononcé comme un monologue après son réveil, et comme s'il voulait prolonger à voix haute et de façon audible pour un cercle d'auditeurs ce qu'il avait vu et pensé pendant son sommeil. Il y avait assurément une logique dans ces phrases, même s'il s'agissait d'une logique entièrement différente de celle qu'Edmund Husserl employait d'habitude avec tant de maîtrise et de précision. Puis il demeura silencieux un moment et il sembla que son âme était revenue de nouveau à son corps depuis l'autre côté. Lorsqu'il remarqua l'infirmière qui se tenait auprès de son lit, il demanda, 
en référence à sa mort qu'il sentait probablement approcher : «Est-il possible aussi de bien décéder?»-L'infirmière : «Oui, dans une paix complète ». - Husserl : « Comment est-ce possible? » - L'infirmière : «En Dieu ». - Husserl : «Vous ne devez pas penser que j'ai peur de la douleur, mais elle me sépare de Dieu ».

Comme il a dû souffrir à l'idée torturante de n'être pas capable de mener sa mission à son terme, de porter en lui une nouvelle œuvre philosophique, une œuvre à venir, de la voir en idée et pourtant de n'avoir plus la force de l'élaborer ! Jusqu'à cet instant, sa vie, sa souffrance et sa préparation à la mort avaient porté le sceau d'un style de vie d'une dignité antique. De fait, on aurait aisément pu dire qu'il faisait face à la mort tel Socrate, seul et sans crainte, que seul ce qui arrivait dans sa mère-patrie l'oppressait lourdement. Mais à présent, le cours de sa vie prit un virage d'une douceur imperceptible, d'une façon d'abord tâtonnante, puis avec toujours plus d'assurance et de clarté, [137] en direction du royaume de la pensée et de la foi chrétiennes. L'infirmière, qui sut se frayer un accès à cette grande âme avec un tact extraordinaire, lui récitait de mémoire le vingt-deuxième Psaume dans la traduction de Luther : «Le Seigneur est mon berger». Au moment où l'infirmière Kläre atteignait les mots : "Passerais-je un ravin de ténèbres, je ne crains aucun mal car Tu es près de moi ", Husserl dit : «Oui, c'est cela, c'est bien ce que je crois. Il devrait être près de moi, mais je ne Le sens pas ». Après quoi, l'infirmière lui récita le cantique : «So nimm denn meine Hände und führe mich [...] Du führst mich doch zum Ziele, auch durch die Nacht » [«Prendsmoi donc par la main et conduis-moi [...] Tu me conduis au but même à travers la nuit »] $]^{21}$ - Husserl : «Oui, c'est ainsi. Que pourrais-je encore vouloir et sentir? Vous devez maintenant prier pour moi ».

Ce Jeudi Saint, vers 21 heures, il dit à sa femme : « Dieu m’a pris en grâce. Maintenant, il m'a autorisé à mourir ». De façon tout à fait significative, il avait adressé ces mots qui émanaient d'un amour conjugal authentique à celle qui avait été sa compagne loyale cinquante années durant. Au cours d'une longue et belle conversation que nous avons eue, elle à mon bras tandis que nous marchions sur la terrasse qui donnait sur la pièce du malade, elle m'avait confié : «Dans notre mariage, je n'ai voulu être que la dalle sur laquelle il marche ». Voilà comment cette femme d'esprit et de caractère résumait la tâche de sa vie aux côtés de ce grand homme.

À partir de ce Jeudi Saint, Husserl ne dit plus un seul mot de son travail philosophique, qui avait pourtant occupé ses pensées tout au long de ces derniers mois. À quel point sa vie entière avait été soumise à la mission confiée par un pouvoir supérieur se révélait seulement alors qu'il allait mourir. À présent, il se sentait enfin déchargé et délivré de sa tâche.

106 Dans les brefs moments qui lui ont encore été donnés avant sa fin dernière, son regard était tourné exclusivement vers Dieu et vers les cieux. C'est à présent qu'on voyait à quel point il s'était toujours tenu dans la grâce, à quel point son âme avait vécu avec le Christ dans un lien intime et profond, même s'il avait recouvert et dissimulé la dimension religieuse dans son existence.

\section{5 avril 1938, Vendredi Saint}

108 L'ultime conversation, l'adieu. Ce matin, à son réveil, sa femme lui dit : « Aujourd'hui, c'est Vendredi Saint». - Husserl : «Quel grand jour, le Vendredi Saint ! Oui, le Christ nous a tous pardonnés ». Il passa la journée entière à dormir, pour l'essentiel de façon 
peu profonde, dans cet étrange demi-sommeil propre aux mourants, puisque l'âme ne cesse de faire des allers-et-retours entre deux mondes.

J'arrive en soirée. Comme je me tiens à son chevet avec Mme Husserl, il lève le bras. Un sourire de reconnaissance anime ses traits. Le moindre mouvement lui cause une souffrance visible. Il n'en saisit pas moins ma main, l'embrasse avec sa galanterie caractéristique et la garde dans la sienne.

110 Alors que nous sommes seuls, il demande, avec une grande difficulté à respirer, à être soutenu, [138] puis il demeura assis, appuyé sur mes bras. Un grand silence s'installe jusqu'à ce qu'il dise doucement, comme dans une plainte: "Nous avons vivement demandé à Dieu qu'il nous permette de mourir. À présent, il nous a accordé cette permission. Mais quelle grande déception d'être toujours en vie». J'essaie de lui communiquer la forte espérance du chrétien en lui disant : «Tel le Christ sur la croix, vous devez vous aussi souffrir, et aujourd'hui encore, jusqu'à la fin». Ce à quoi il répond, avec une conviction profonde et un grand sérieux - on aurait dit un "Amen » : «Oui ». Puisque je le vois plein d'agitation intérieure et d'angoisse sans être capable de parler, je lui dis : « Dieu est bon ; oui, Dieu est si bon ». - Husserl : « Dieu est bon, oui, mais si incompréhensible. C'est maintenant une grande épreuve pour nous ».

111 Puis il semble chercher quelque chose. Avant même qu'il continue à parler, ses mains bougent tandis que l'expression de son visage était celle d'une concentration profonde, comme s'il ne cessait de prier sans parole. Finalement, illustrant de ses mains les mots qu'il cherchait, il dit: «Deux mouvements se cherchent constamment l'un l'autre, se trouvent et se cherchent à nouveau ». Je tente d'élever ses mots dans la dimension surnaturelle et de leur donner un sens chrétien : « Oui, le Ciel et la Terre se rencontrent en Jésus. Dans le Christ, Dieu s'est approché de l'homme ». - Husserl (avec animation) : "Oui, c'est cela. Il est l'analogie entre... ». Comme il cherche ses mots et qu'il est visiblement tourmenté par le fait de ne pas parvenir à les trouver, j'essaie encore de mener à son terme la ligne de pensée qui avait commencé : "Oui, Jésus s'est fait l'analogie entre Dieu et nous autres humains. C'est cela, le Vendredi Saint: la rédemption et Pâques en même temps ». - Husserl (comme s'il était soulagé et libéré, avec une profonde conviction et un regard, complètement détaché du monde, de compréhension intime - un regard qui m'émut profondément) : « Oui, c'est cela ».

112 Après un moment - il s'était déjà allongé sur son oreiller depuis quelque temps - il agite de nouveau les mains et dessine des lignes dans l'air, il fait également des gestes défensifs, comme s'il voyait quelque chose qui l'effrayait. En réponse à ma question de savoir ce qu'il pouvait bien voir, il répond comme absorbé dans une pensée de la profondeur d'un rêve, et d'une voix qui m'est totalement inconnue, qui semble venir de l'au-delà : "Lumière et obscurité, oui, beaucoup d'obscurité, puis de nouveau la lumière... ».

113 Ce fut la dernière conversation de Husserl, comme sa femme me le confirma plus tard. À partir de cet instant, il demeura allongé en silence et dormit la majeure partie du temps. L'un des tout derniers jours, dans l'après-midi, après avoir dormi, il s'exclama à son réveil, le regard radieux et les yeux brillant intensément: «Oh, j’ai vu quelque chose de si merveilleux! écrivez, vite!». Avant que l'infirmière ait pu mettre la main sur le bloc, il avait déjà défailli de faiblesse. Il emporta le secret de cette vision avec lui dans l'éternité, là où la Vérité éternelle ne tarderait pas à l'approcher, lui l'infatigable chercheur de la vérité. Il mourut le 27 avril $1938^{22}$. 
En guise de complément, nous proposons ci-dessous la traduction d'une lettre inédite rédigée par Adelgundis Jaegerschmid le lendemain de la mort de Husserl. Une copie de cette lettre se trouve aux Archives Jean Héring, à l'Université de Strasbourg; nous remercions Édouard Mehl de nous l'avoir transmise.

Fribourg, Sainte Lioba

$117 \quad 28$ avril 1938

118 Chère Maria,

Je souhaiterais te faire part, à toi, du décès vraiment comblé de grâces qui fut celui de notre maître révéré et bien-aimé.

Husserl était malade depuis le mois d'août 1937; il a souffert indiciblement avant de parvenir à un tel accomplissement et à une telle transfiguration. C'est hier matin, à $5 \mathrm{~h} 45$, que Dieu l'a rappelé à Lui ; je suis certaine qu'il a accédé directement à la lumière de la Vérité éternelle, qu'il a cherchée toute sa vie si fidèlement avec tant de responsabilité. Pendant les cinq semaines qui ont précédé, j’ai encore passé quelques nuits de garde à ses côtés, au cours desquelles il parla d'une manière bouleversante de sa philosophie, la profonde communauté spirituelle qu'il y avait entre nous lui faisant oublier que ses autres étudiants étaient scientifiquement bien plus de son niveau que moi. Je ne peux pas te dire, chère Maria, quel trésor de bonté, d'amitié, d'amour et de la plus tendre communion notre bon maitre m'a offert durant ces dernières semaines et jusqu'à peu de temps avant sa mort. Jusqu'au bout, il m'a clairement reconnue et m'a fait part de sa joie, y compris par signes lorsqu'il ne pouvait plus parler. Environ deux semaines avant sa mort - à partir de Vendredi Saint - il s'est complètement détaché de son travail (qui lui tenait jusqu'alors encore tellement à cœur) et a confié toute son âme à Dieu. L'après-midi de ce Vendredi Saint, il m’a dit: "nous avons demandé sincèrement à Dieu qu'il nous permette de mourir et il nous a accordé cette permission; mais quelle déception de devoir encore attendre ». Je lui ai parlé de la bonté de Dieu, du Christ et de la rédemption. Il a répondu avec une conviction profonde : «Oui, c'est ainsi ». Il se tenait déjà là-bas dans l'attente et ne revenait vers nous que pour y rencontrer la souffrance, l'angoisse passagère. Il lui fallait boire le calice du Christ, car il était vraiment tout à Lui. "J'ai vu quelque chose de si merveilleux », dit-il un jour à son réveil, illuminé et radieux, «écrivez, vite!». Mais déjà, sa tête s'était de nouveau inclinée. À la fin, il lui restait encore l'inquiétude que la souffrance puisse le séparer de Dieu, et celle de savoir où il en arriverait s'il cessait de penser, de savoir s'il serait encore auprès de Dieu. S'il n'y avait cette douloureuse absence dans ma vie personnelle, je devrais exulter; mais Dieu comprendra bien. Le christianisme, la condition humaine, la communion des saints, le rapport à Dieu en deuxième personne, la bonté, l'amour, le devoir - tout cela a reçu pour moi grâce à Husserl un sens profond et renouvelé, tout est en tout cas plus vaste et plus grand que ce qui est donné à lire dans ce qui est imprimé. Être étudiant de Husserl oblige, nous le savons bien. Son testament, pour ainsi dire, est pour moi cette parole qu'il eut lorsque, à l'automne, nous étions dans son bureau, assis main dans la main et que la flèche de la cathédrale se dressait devant nous, solennelle et magnifique: "Oh, qu'il vous soit donné de gagner dans l'amour des âmes à l'amour ", par quoi il pensait avant tout à la jeunesse à venir. La solitude se fait autour de nous, tout devient plus sombre et plus 
lumineux à la fois. Des quatre professeurs de ma promotion, seul Finke, âgé de 82 ans, est encore parmi nous ; Husserl, Göller et Klinge sont morts.

Mme Husserl habite au 6 Schöneckerstrasse. Elli, sa fille dont le mari est devenu Professeur à l'université de Harvard, a passé deux mois ici mais n'a pas pu rester jusqu'à la fin. Gerhard, le juriste, donne cours en ce moment dans une université de Virginie et n'a pas pu venir une seule fois.

Bien à toi, dans la pensée de celui qui fut pour nous le guide de notre jeunesse.

Sœur Adelgundis.

\section{NOTES}

1. R. Rolland, Mahatma Gandhi, Paris, Stock, 1924 - NdT.

2. En théologie scolastique, cette expression désigne le caractère objectif du sacrement ou de l'effet qui en dérive, par opposition aux dispositions subjectives de la personne qui le reçoit ou à travers lesquelles elle le met en œuvre, et qui sont alors nommées opus operans (ou operantis).

3. Kulturkampf. Cette expression renvoie à l'ensemble des tensions politiques et diplomatiques par lesquelles s'est traduite, entre 1870 et 1887, l'opposition entre la Prusse puis l'Empire allemand et l'Église catholique allemande. Le terme désigne en particulier la politique libérale et les dispositions juridiques par lesquelles le pouvoir politique a cherché à priver l'Église catholique de toute influence sociale.

4. Il s'agit du concordat signé le 20 juillet 1933 entre le Saint-Siège et le III ${ }^{\mathrm{e}}$ Reich.

5. Cf. supra, note 3 .

6. Ces deux conférences, intitulées respectivement «La philosophie dans la crise de l'humanité européenne » et «La psychologie dans la crise de la science européenne » forment le point de départ des réflexions qui aboutiront au dernier grand ouvrage de Husserl, qui ne fut que partiellement publié de son vivant, La Crise des sciences européennes et la phénoménologie transcendantale. Notons que contrairement à ce que Husserl indique quelques lignes plus loin, c'est bien au printemps qu'il donnera finalement sa conférence à Vienne, les 7 et 10 mai. Quant au voyage à Prague, il aura effectivement lieu à la mi-novembre 1935.

7. Eugen Fink devint l'assistant de Husserl à la fin de l'année 1928, avant de devenir son collaborateur à partir de 1930, c'est-à-dire un interlocuteur d'une proximité telle avec la pensée de Husserl que ce dernier alla jusqu'à lui confier la conception et la rédaction de nombreux paragraphes, aussi bien dans le cadre de la reprise des Méditations cartésienne (et notamment la célèbre Sixième Méditation cartésienne) que dans celui de l'élaboration de ce qui devait devenir la Crise des sciences européennes et la phénoménologie transcendantale. Pour une reconstruction détaillée des années de collaboration de Fink avec Husserl, cf. R. Bruzina, Edmund Husserl \& Eugen Fink. Beginnings and Ends in Phenomenology. 1928-1928, New Haven \& London, Yale University Press, 2004, chap. 1, p. 1-73.

*. ${ }^{*}$ En français dans le texte.

8. Cf. supra, note 6 .

9. C'est à partir de 1926 qu'encouragée par le père Erich Przywara, Edith Stein s'attelle à la traduction allemande des Quaestiones disputatae de veritate de saint Thomas, premier acte de la confrontation qu'elle mènera patiemment entre la philosophie thomiste et la phénoménologie. 
10. R. Guardini, L'Univers religieux de Dostoïevski [1932], trad. par H. Engelmann et R. Givord, Paris, Seuil, 1947.

11. R. Guardini, Le Seigneur : méditations sur la personne et la vie de Jésus-Christ (1936), trad. par le R. P. Lorson, Paris, Alsatia, 1945.

12. H. Ball, Byzantinisches Christentum : Drei Heiligenleben, Munich-Leipzig, Duncker \& Humblot, 1923.

13. R. Guardini, Im Spiegel und Glechnis - Bilder und Gedanken, Mayence, Mathias Grünewald Verlag, 1932.

14. Il s'agit des deux premières parties de l'ouvrage que nous connaissons aujourd'hui sous le titre La Crise des sciences européennes et la phénoménologie transcendantale, publiées initialement par Arthur Liebert à Belgrade dans le premier numéro de la revue Philosophia (1936).

15. Nous corrigeons ici le texte original, qui écrit relevata.

16. W. Windelband, Einleitung in die Philosophie. Grundriß der philosophischen Wissenschaften, Bd. 1., Tübingen, 1914. La référence à Paulsen est incertaine.

17. Il s'agit du IX Congrès International de Philosophie, qui eut lieu à Paris du 31 juillet au 6 août 1937, où furent données plus de 300 communications et dont les actes ont été publiés quelques mois plus tard par l'éditeur Hermann, en 12 volumes.

18. Il s'agit de la Brockhaus Enzyklopädie [Encyclopédie Brockhaus], qui connaît entre 1928 et 1935 sa quinzième édition (en vingt volumes, un supplément et un atlas), sous le titre Der Große Brockhaus. 19. A. Stifter, Witiko, 3 vol., 1865-1867.

20. Cf. supra, note 6 .

21. Célèbre cantique de Julie Katharina von Hausmann, extrait de Maiblumen: Lieder einer Stillen im Lande, 4. Auflage, Bd. I, Berlin, Eduard Beck, 1862, p. 66-67.

22. Nous corrigeons ici le texte original qui, de façon surprenante, indique fautivement la date du 26 avril. 